\title{
An application of advanced technology in disaster management
}

\author{
Dr. Gopal Krishna, MBBS,MD,DIH \\ Vice-Principal \& Head of Department of Community Medicine, College of Medical Sciences \& \\ Managing Editor, Journal of College of Medical Sciences, Bharatpur, Nepal
}

Disaster is a catastrophic event in which a large numberof people,flora and fauna are affected all of sudden at one time causing premature deaths,impaired health status and diminished quality of life. To give importance to disaster reduction 1990's was designated as the international decade of natural disaster reduction. When ever geological or climatological events that threaten people or property are termed as natural hazards and when they destroy people's lives and livelihoods it is termed as Natural disaster.

Most destructive disasters are climatological and geological. Climatological disasters are more frequent than Geological ones whether measured by economic loss or by deaths or injuries Asia is most prone to Natural disasters followed by Latin America and least prone countries are Europe, North America and Australia. For each major natural disaster in Europe and Australia there are 10 in Latin America an, Africa and 15 in Asia.

Disasters may occur as sudden or they may have insidious onset .Injuries exceed deaths in storms, fire, explosions while deaths exceed injuries in earthquake, landslide, volcanic eruptions and floods. Theremay be impaired health due to injuries, emotional stress, epidemics and increase in indigenous diseases due to disruption in health services. The long term effects associated with climatological disasters are increase in infectious diseases and toxic effects. Most important cause of death is drowning in water based disasters and inland based disasters mostly injuries. Death in such cases is due to physical trauma.

The management process involves mitigation, preparedness, response and recovery in the disaster cycle. Mitigation is to reduce or eliminating risk by applying long term measures. In other words limiting the impact of disaster by applying structural and non structural interventions e.g. layout of building to resist disaster, installation of earthquake valves, seismic retrofits of property and securing items inside the building. Properties should not be purchased which are exposed to hazards i.e. in area of landslide and floodplains.

Next comes Preparedness which is the planning for shelter, installations of warning system, communication plans, stockpiling, inventory and maintaining disaster supplies, Disaster supply kits-for evacuation and survival kits which helps to survive at least $72 \mathrm{hrs}$. It contains medicine, food, flashlights, candles and money, preparing community response teams and over and all casualty prediction.

Preparedness phase is followed by Response phase which calls for emergency services for search and rescue..These efforts should be made at an early stage.

Recovery phase starts after the immediate threat to human lives subside. Restoring an area often through rebuilding and rehabilitation -then returning to mitigation phase.

During the process of management situations are often anticipated and planned for in hospitals and emergency care systems sorting of victims according to the severity of injury or illness and assigning priorities of treatment during triage rounds. The triage is a French word meaning to pick out or sort. It has two components -Sorting of victims according to severity of illness or injuries and assigning priorities for treatment. 
Developed countries have shown that although science and technology cannot prevent Natural disasters from happening, it can dramatically reduce the impact in terms of human suffering.

Developing countries suffer more than $95 \%$ of all deaths caused by natural disasters. Factors responsible for such high mortality is their high population densities and poor infra structure coupled with unstable land forms and exposure to severe weather events.

The most advanced technology in disaster management is the Remote Sensing. Its uses are many. It can be used for risk modelling and vulnerability analysis. It is very specific for early warning and even damage assessment but for that you need a very skilled personnel for data analysis and its interpretation. Two complementary types of satellites are relevant to disaster management as many types of satellites are used for earth observation. Polar orbiting satellite often fly in relatively low in Sensors are fixed in satellite orbit around $1000 \mathrm{kms}$ above ground providing high special resolution but the data is collected over same point once every few days. The other type of satellite -Geostationary are positioned at an altitude of $36000 \mathrm{kms}$ which orbit the earth at the same speed as earth rotates on its axis. It collects data every 15 minutes at same point. These satellites give more accurate, frequent and almost instantaneous data over large areas anywhere in the world. Since one or more sensors are fixed in satellites on board it take measurement in different wavelengths. They are very useful in disaster monitoring e.g. the Infrared sensor can pick up flood and for spotting active fire there are Thermal sensors. Other Microwave sensors have the quality to penetrate the clouds and smoke and can be used to measure deformations in earth before and after volcanic eruptions and earthquakes.

The Remote sensing is being used by developed countries to monitor and manage disasters. Japan has invested a lot in installation of an earthquake warning system that detects the waves that are given off by earthquakes. Once detected the computers register and broadcast warnings via television and cell phones. The system automatically shut down energy and industrial facilities and transportation services.

Tsunami was the aftermath of initial quake on 11March 2011 which struck the North East coast of Japan triggering a 10 meters Tsunami that swept away every thing in its path including houses and cars and gashed and displaced a 150 mile long and 50 mile wide areas of ocean crust according to US Geological Survey. Hundreds of miles away many people working in Tokyo skyscrapers knew that earthquake was coming. This was the biggest earthquake to hit Japan in 140 years.

We have access to ready processed data which are increasingly made freely available. Cheap and users friendly Geographical information system can also help integrate relevant local and regional data .we have many skilled personnels to analyse and use Remote Sensing data. The mainstay in planning and management for disasters is the utilisation of such data in collaboration with emergency management framework with careful risk approach and threat assessment.

\section{References}

1.http://www.emergencymgmt.com/disaster/japan-tsunami-tsunami warning system

2.http://www.bt.cdc.gov/disaster/tsunami/health effects.asp

3. http://www.scidev.net

4. http://books.google,co,in/epidemiology of tsunam

5.Sian Lewis,Sc idev.n et 